\title{
Gliding Basal Cell Migration of the Urothelium during Wound Healing
}

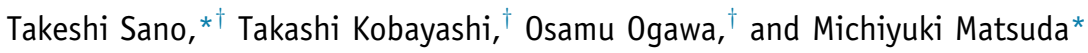

From the Departments of Pathology and Biology of Diseases* and Urology, ${ }^{\dagger}$ Graduate School of Medicine, Kyoto University, Kyoto, Japan

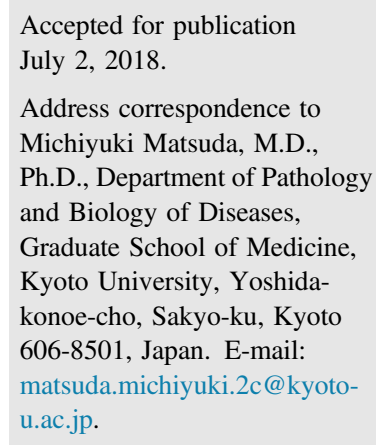

Address correspondence to Michiyuki Matsuda, M.D., Ph.D., Department of Pathology and Biology of Diseases, Graduate School of Medicine, Kyoto University, Yoshidakonoe-cho, Sakyo-ku, Kyoto 606-8501, Japan. E-mail: matsuda.michiyuki.2c@kyotou.ac.jp.

\begin{abstract}
Collective cell migration during wound healing has been extensively studied in the epidermis. However, it remains unknown whether the urothelium repairs wounds in a manner similar to the epidermis. By in vivo two-photon excitation microscopy of transgenic mice that express fluorescent biosensors, we studied the collective cell migration of the urothelium in comparison with that of the epidermis. In vivo time-lapse imaging revealed that, even in the absence of a wound, urothelial cells continuously moved and sometimes glided as a sheet over the underlying lamina propria. On abrasion of the epithelium, the migration speed of each epidermal cell was inversely correlated with the distance to the wound edge. Repetitive activation waves of extracellular signal-regulated kinase (ERK) were generated at and propagated away from the wound edge. In contrast, urothelial cells glided as a sheet over the lamina propria without any ERK activation waves. Accordingly, the mitogen-activated protein kinase/ERK kinase inhibitor PD0325901 decreased the migration velocity of the epidermis but not the urothelium. Interestingly, the tyrosine kinase inhibitor dasatinib inhibited migration of the urothelium as well as the epidermis, suggesting that the gliding migration of the urothelium is an active, not a passive, migration. In conclusion, the urothelium glides over the lamina propria to fill wounds in an ERKindependent manner, whereas the epidermis crawls to cover wounds in an ERK-dependent manner. (Am J Pathol 2018, 188: 2564-2573; https://doi.org/10.1016/j.ajpath.2018.07.010)
\end{abstract}

Collective cell migration is observed in many physiological and pathologic processes, such as development, epithelial wound healing, and cancer cell invasion. ${ }^{1-3}$ In epithelial wound healing, monolayer or stratified layers of the epithelial cells migrate as a group and close the tissue defect over the underlying lamina propria. ${ }^{4-6}$ Various physical and chemical cues induce front-to-rear polarity of cells at the free edge of a wound, referred to as leader cells, and their migration toward the free space. ${ }^{3,6}$ The leader cells transmit the polarity to the follower cells through mechanical coupling mediated by cell-cell junctions and organize a movement in groups. ${ }^{7}$ Moreover, diffusible molecules, such as $\mathrm{Ca}^{2+}$, hydrogen peroxide, ATP, and growth factors, also contribute by transmitting signals to the follower cells during the collective cell migration. ${ }^{8}$

The molecular basis underlying the coordinated epithelial cell migration during wound healing has been extensively studied in vitro by using epithelial cell lines, including MDCK cells. For example, the Rho-family GTPases and extracellular signal-regulated kinase (ERK) have been shown to play roles in this migration process in MDCK cells. ${ }^{9,10}$ The classic scratch method to generate a wound in the monolayer MDCK cell sheet not only opens the space but also damages the cells, generating reactive oxygen species. It has been proposed that reactive oxygen species at the wound edge are required for the activation of ERK and cell migration. ${ }^{11}$

A new window into the study of the wound healing process has been opened by biosensors based on Förster resonance energy transfer (FRET). ${ }^{12-14}$ For instance, activation of Rho-family GTPases in the leader cells has been demonstrated by the time-lapse FRET imaging of wounded MDCK cell monolayers. ${ }^{15,16}$ More recently, we have

\footnotetext{
Supported by JSPS KAKENHI grants $15 \mathrm{H} 02397$, 15H05949, and 16H06280, JST CREST grant JPMJCR1654, the Nakatani Foundation (M.M.), and JSPS grant-in-aid for young scientists (A) 25713055 (T.K.).

Disclosures: None declared.
} 
discovered that repetitive waves of ERK activation were propagated away from the wound edge not only in MDCK cells but also in the ear skin of mice. ${ }^{17,18}$ Tidal waves of ERK activation were previously found to be propagated from the wound edge by immunohistochemistry ${ }^{10,11}$; however, the repetitive waves of ERK activation from the wound edge ${ }^{17}$ or spontaneous wavelets in the regions apart from the wound edge could only be visualized by time-lapse imaging of ERK activity with FRET biosensors. ${ }^{18}$ More important, cells migrate against the direction of the ERK activation wave in both the mouse epidermis and the MDCK monolayer sheet. ${ }^{18}$

A substantial part of our knowledge about wound healing in vivo comes from studies of epidermal wound healing, and the basic mechanism underlying wound healing is assumed to be conserved among different animals and tissues.,19 Meanwhile, although there have been several studies on the wound healing of the urothelium, ${ }^{20-24}$ it remains elusive whether urothelial wounds are repaired in the same way as epidermal wounds. Recently, we performed in vivo imaging of the mouse urothelium by two-photon excitation microscopy (TPEM). ${ }^{25}$ During the course of the study, we noticed that the urothelium sometimes glides over the underlying lamina propria, and this observation urged us to examine the collective migration of the urothelium during wound healing. Herein, we demonstrate that the collective migration of the urothelium is significantly different from that of the epidermis, not only regarding the mode of migration but also in terms of the requirement for ERK activity.

\section{Materials and Methods}

\section{Ethical Approval}

The animal protocols were reviewed and approved by the Animal Care and Use Committee of Kyoto University Graduate School of Medicine (Kyoto, Japan; numbers 12064, 13074, 14079, and 15064).

\section{Animals}

Transgenic mice expressing ERK FRET biosensors have been described previously. ${ }^{26}$ ERK FRET biosensors, EKAREV-nuclear export signal and EKAREV-nuclear localization signal, are localized in the cytoplasm and the nucleus, respectively. ${ }^{26}$ EKAREV-nuclear export signal and EKAREV-nuclear localization signal that were backcrossed more than five generations to C57BL/6N Jcl (CLEA Japan, Tokyo, Japan) were used for analysis. The Fucci mice, which express mAG-hGeminin (1/110) and mKO2hCdt1 (30/120), were obtained from the Laboratory for Animal Resources and Genetic Engineering, RIKEN Center for Developmental Biology. ${ }^{27}$ Mice were housed in a specific pathogen-free facility in temperature-controlled rooms with a 14-hour light/10-hour dark cycle and received a routine chow diet and water ad libitum. For intravital imaging of the skin and the bladder, 12- to 25week-old mice were used. At the end of the experiments, mice were euthanized by anesthetic overdose.

\section{Two-Photon Excitation Microscopy}

We used an FV1000MPE-BX61WI upright microscope (Olympus, Tokyo, Japan) equipped with a $25 \times / 1.05$ waterimmersion objective lens (XLPLN 25XWMP; Olympus) and an InSight DeepSee Ultrafast laser $(0.95 \mathrm{~W}$ at $900 \mathrm{~nm}$; Spectra Physics, Mountain View, CA). The excitation wavelength for cyan fluorescent protein (CFP) was $840 \mathrm{~nm}$. An infrared light-cut filter, BA685RIF-3 (Olympus); two dichroic mirrors, DM505 and DM570 (Olympus); and four emission filters, FF01-425/30 (Semrock, Rochester, NY) for the second harmonic generation, BA460-500 (Olympus) for CFP, BA520-560 (Olympus) for yellow fluorescent protein, and 645/60 (Chroma Technology, Bellows Falls, VT) for Qtracker 655 (Life Technologies, Carlsbad, CA), were used. Qtracker 655 is intravenously administered with other reagents to confirm drug delivery to target organs. For Fucci mouse imaging, an IR-cut filter, RDM690 (Olympus); two dichroic mirrors, DM505 and DM570; and three emission filters, FF01-472/30 (Semrock) for second harmonic generation images, BA495-540 (Olympus) for $\mathrm{mAG}$, and BA575-630 (Olympus) for $\mathrm{mKO} 2$, were used. The microscope was equipped with a two-channel GaAsP detector unit and two built-in photomultiplier tubes. FluoView software version 4.1a (Olympus) was used to control the microscope and to acquire images, which were saved in the multilayer 16-bit tagged image file format.

\section{Intravital Imaging of Mouse Tissues}

Intravital imaging of the bladder was performed, as described previously. ${ }^{25}$ Briefly, female mice were anesthetized by inhalation of $1 \%$ to $1.5 \%$ isoflurane (Abbott Laboratories, North Chicago, IL) and placed in the supine position on an electric heat pad maintained at $37^{\circ} \mathrm{C}$. A 24-gauge ethylene tetrafluoroethylene catheter (Terumo, Tokyo, Japan) connected to a 50-mL bottle of normal saline (Otsuka Pharmaceutical Factory, Tokushima, Japan) was inserted transurethrally into the bladder. The intravesical pressure was controlled by the bottle's height and kept at 15 to $20 \mathrm{~cm} \mathrm{H}_{2} \mathrm{O}$ for 30 minutes. Then, the catheter, which caused mechanical irritation and intensified the rhythmic muscle contraction of the bladder, was removed for stable long-term imaging. The bladder was pulled out of the abdominal cavity, and the bladder wall was immobilized on a custom-made vacuum-stabilized imaging window (Olympus). For multidimensional imaging of the urothelium and the underlying lamina propria, Z-stack images were acquired using a 2.4 digital zoom at $0.5-\mu \mathrm{m}$ intervals and at a scan speed of 8 microseconds/pixel. CFP, Qtracker 655, and second harmonic generation were imaged to show cells, blood vessels, and collagen fibers, respectively. Time-lapse 

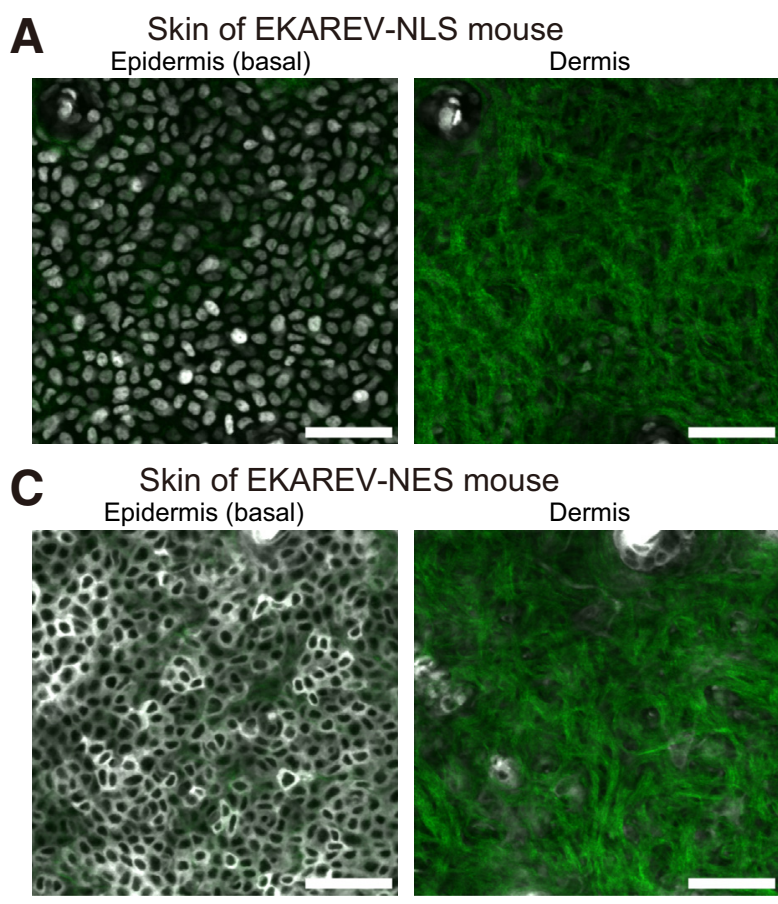

E

Motion analysis

Epidermis

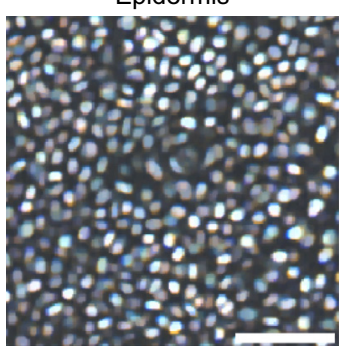

Time (minutes) 0

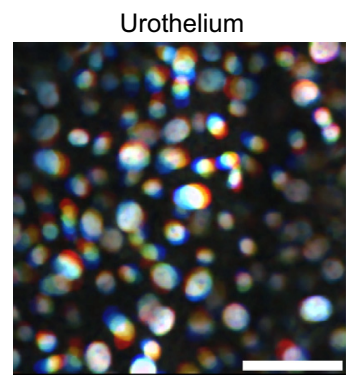

60

G

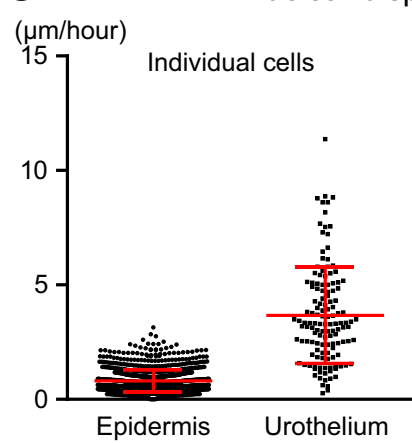

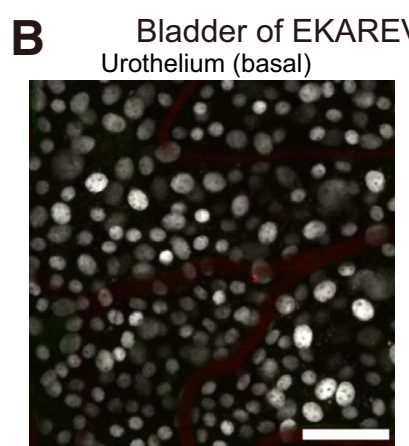

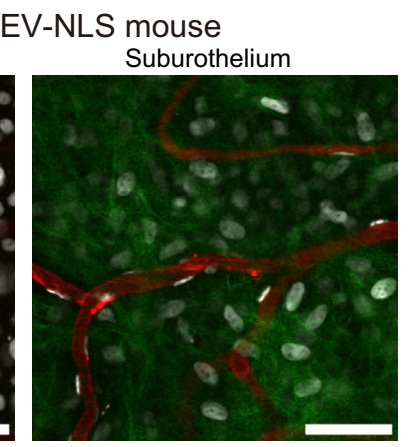

D

Bladder of EKAR
Urothelium (basal)
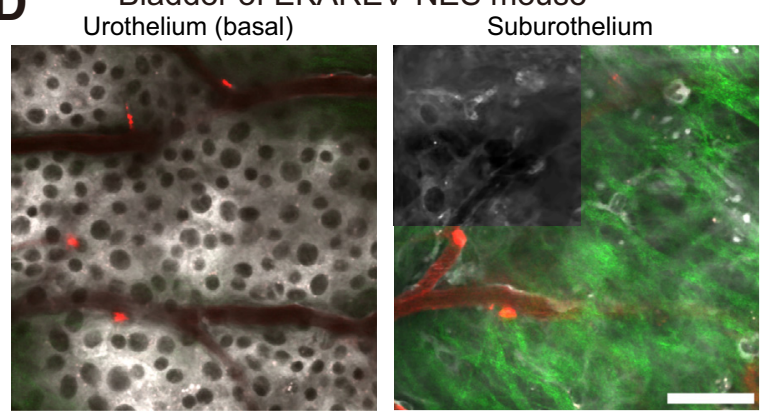

F Trajectory of nuclear centroid
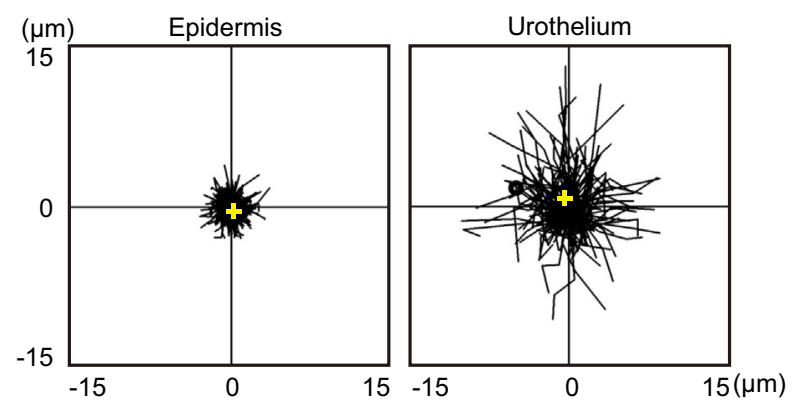

H Motion analysis

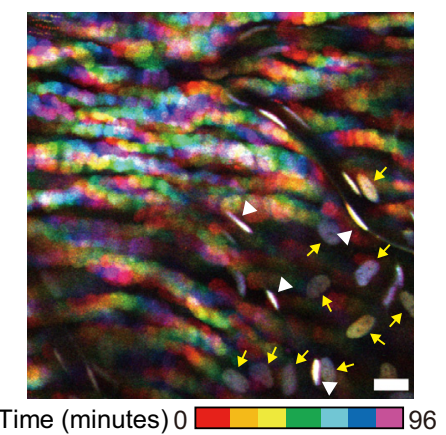

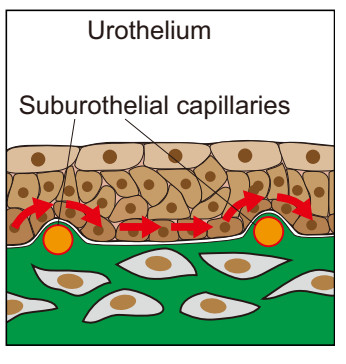

Figure 1 Gliding of the urothelium over the lamina propria. A-D: Merged images of Förster resonance energy transfer (FRET; gray), second harmonic generation (SHG; green), and Qtracker 655 (red) by multiphoton imaging of the mouse ear skin and bladder wall expressing EKAREV-nuclear localization signal (NLS; A and B) and EKAREV-nuclear export signal (NES; C and D). D: The upper left quarter of SHG of the suburothelium is removed to show the interstitial cells more clearly. E and F: Motion (E) and trajectory (F) analysis of the epidermis and the urothelium (Supplemental Video S1) of an EKAREV-NLS mouse. E: FRET images acquired every 20 minutes are superimposed and four colors are assigned according to the time points of image acquisition, as indicated by the color bar. F: Displacement of the centroid of the nuclei during the 1-hour imaging is shown on X-Y planes. The yellow crosses indicate the averages. G: Bee-swarm plots of the displacement of the nuclei for all cells and averages. $\mathrm{H}$ : Left panel: A superimposition of 30 time-series FRET images at the interface between the urothelium and the lamina propria. Seven colors are assigned according to the time points of image acquisition, as indicated by the color bar. All images were superimposed to show that the urothelial cells moved from left to right as the time elapsed. The elliptical nuclei of the interstitial cells (arrows) and the elongated nuclei of the endothelial cells (white arrowheads) are white, indicating that these cells stayed in the same position during the imaging (Supplemental Video S2). Right panel: A schematic diagram of the urothelium gliding over the lamina propria. The red arrows illustrate the track of basal cells gliding over the lamina propria. Data are expressed as means \pm SD (G). $n>1000$ epidermises (F); $n>100$ urothelia (F). Scale bars: $50 \mu \mathrm{m}(\mathbf{A}-\mathbf{E}) ; 20 \mu \mathrm{m}(\mathbf{H})$. 
images were acquired every 5 or 6 minutes using a 1.2 to 2.4 digital zoom at a scan speed of 4 microseconds/pixel.

For the wound healing analysis of the urothelium, a square $100 \mu \mathrm{m}$ on each side was set under the two-photon

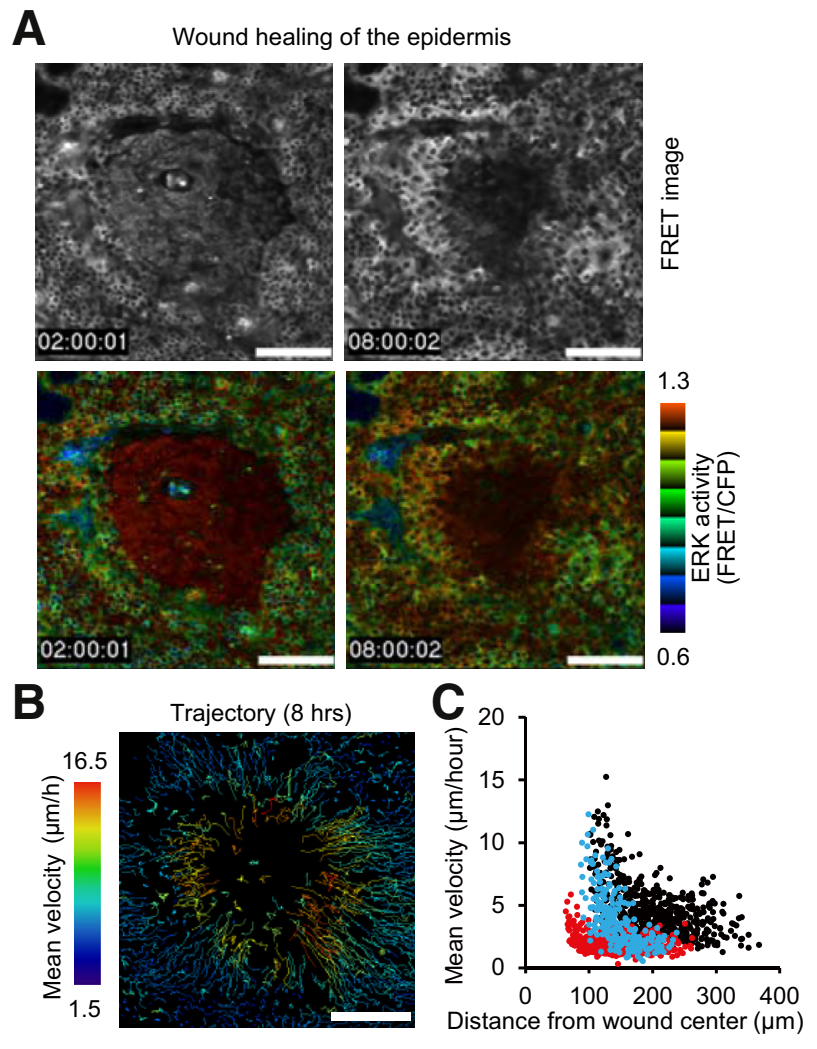

D
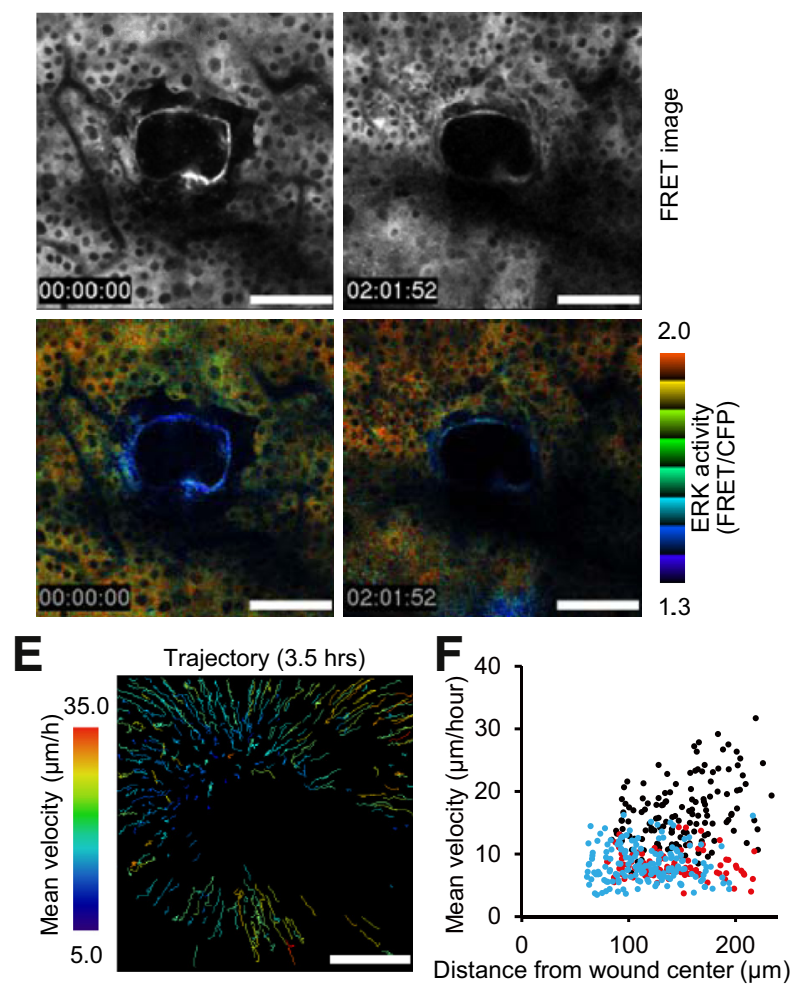

excitation microscope. After increasing the laser power to $80 \%$ to $100 \%$, the area was repeatedly scanned until the CFP fluorescence signal became undetectable, even with the highest sensitivity of the GaAsP detector.

Intravital imaging of the ear skin was performed, as described previously. ${ }^{17}$ Hair was removed from an ear by using depilation cream 24 hours before experiments. An ear of an anesthetized mouse was sandwiched between a cover glass and a thermal conductive silicon gum sheet. For multidimensional imaging of the epidermis and underlying lamina propria, Z-stack images were acquired using a 3.0 digital zoom at $0.5-\mu \mathrm{m}$ intervals and at a scan speed of 8 microseconds/pixel. Time-lapse images were acquired every 10 or 12 minutes. An epithelial wound was generated at the ear skin with a 29-gauge needle (Terumo) 2 hours before imaging.

PD0325901 (5 mg/kg), a mitogen-activated protein kinase (MAPK)/ERK kinase inhibitor (EMD Millipore, Billerica, MA), was dissolved in $0.2 \mathrm{~mL}$ phosphate-buffered saline supplemented with $4 \mu \mathrm{L}$ Qtracker 655 and injected via the tail vein at a dose of $5 \mathrm{mg} / \mathrm{kg}$. Dasatinib, a tyrosine kinase inhibitor (AdooQ BioScience, Irvine, CA), was dissolved in $0.15 \mathrm{~mL}$ propylene glycol supplemented with $4 \mu \mathrm{L}$ Qtracker 655 and injected via the tail vein at a dose of $10 \mathrm{mg} / \mathrm{kg}$.

\section{Image Processing}

Microscopic images were analyzed, as described previously, with MetaMorph software version 7.10.1.161 (Molecular Devices, Sunnyvale, CA). ${ }^{28}$ In brief, yellow fluorescent protein images obtained by the excitation of CFP were used as FRET images. The FRET level is evaluated by the FRET/CFP ratio and represented as an intensity-modulated display or golden pseudocolor images. In the intensity-modulated

Figure 2 Distinct mode of collective cell migration between the epidermis and urothelium. A: An epithelial wound was generated on the ear skin of each transgenic mouse expressing EKAREV-nuclear export signal (NES). Two hours after wounding, the mice were observed under twophoton excitation microscopy for 12 hours. Cyan fluorescent protein (CFP) and Förster resonance energy transfer (FRET) images were acquired every 10 minutes to generate video of FRET/CFP ratio images (Supplemental Video S3). Shown here are the FRET image and FRET/CFP ratio image in intensity-modulated display mode with the ratio range shown on the right. B: Tracks and the velocities of the epidermal cells were generated as described in Materials and Methods. C: Cells that could be tracked for at least seven sequential time-lapse images were analyzed to plot the distance from the center of the wound and mean velocity. D: Transgenic mice expressing EKAREV-NES were subjected to observation. Under a two-photon excitation microscope, a urothelial wound was generated by laser ablation. Images were acquired every 6 minutes for 8 hours (Supplemental Video S4). FRET and FRET/CFP ratio images are shown. E: Tracks and the velocities of the epidermal cells were generated as described in Materials and Methods. F: Cells that could be tracked for at least six sequential time-lapse images were analyzed to plot the distance from the center of the wound and mean velocity. The black, cyan, and red dots in $\mathbf{C}$ and $\mathbf{F}$ indicate data sets from three independent experiments. $n=3(\mathbf{C}$ and $\mathbf{F})$. Scale bars $=100 \mu \mathrm{m}$ (A, B, D, and E). ERK, extracellular signal-regulated kinase. 

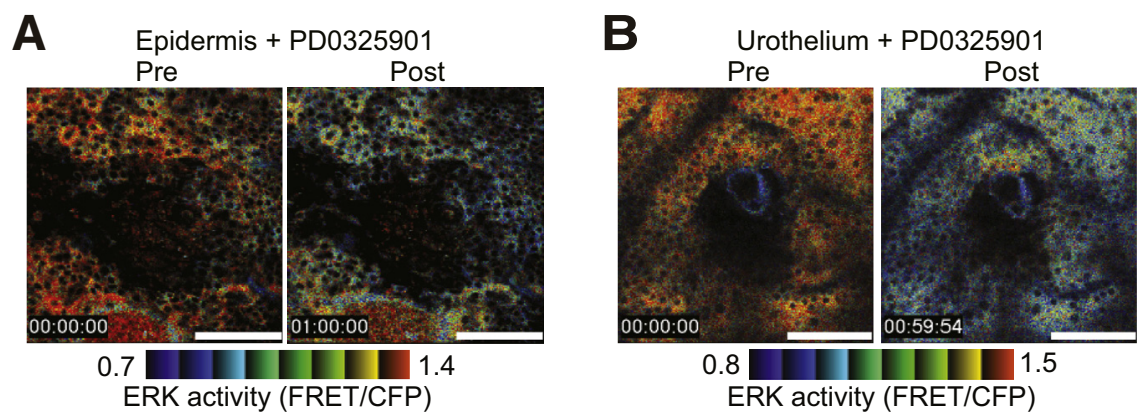

Figure 3 Differential sensitivity to a mitogen-activated protein kinase/extracellular signal-regulated kinase (ERK) kinase (MEK) inhibitor between the wound healing of the epidermis and urothelium. A and B: An epithelial wound or a urothelial wound was generated in each transgenic mouse expressing EKAREV -nuclear export signal. Two hours after wounding, the mice were observed by twophoton excitation microscopy for at least 3 hours. Cyan fluorescent protein (CFP) and Förster resonance energy transfer (FRET) images were acquired to generate FRET/CFP ratio images. The MEK inhibitor PD0325901 $(5 \mathrm{mg} / \mathrm{kg})$ was intravenously injected 1 hour after the start of image acquisition. Representative FRET/CFP ratio images before (Pre) and 1 hour after (Post) the administration of the MEK inhibitor are shown. C: The mean ERK activity (FRET/CFP) of three mice before (Pre) and 1 hour after (Post) the administration of the MEK inhibitor. D: Leader cells within $20 \mu \mathrm{m}$ of the wound edge were subjected to trajectory analysis to calculate the mean velocity of migration. $\mathbf{E}$ and $\mathbf{F}$ : Similar experiments were performed, except that the tyrosine kinase inhibitor dasatinib $(10 \mathrm{mg} / \mathrm{kg}$ ) was administrated at time zero. Three mice were used for each condition. ${ }^{*} P<0.05$, $* * P<0.01$, and $* * * P<0.001$. Scale bars $=100 \mu \mathrm{m}(\mathbf{A}$ and $\mathbf{B})$.
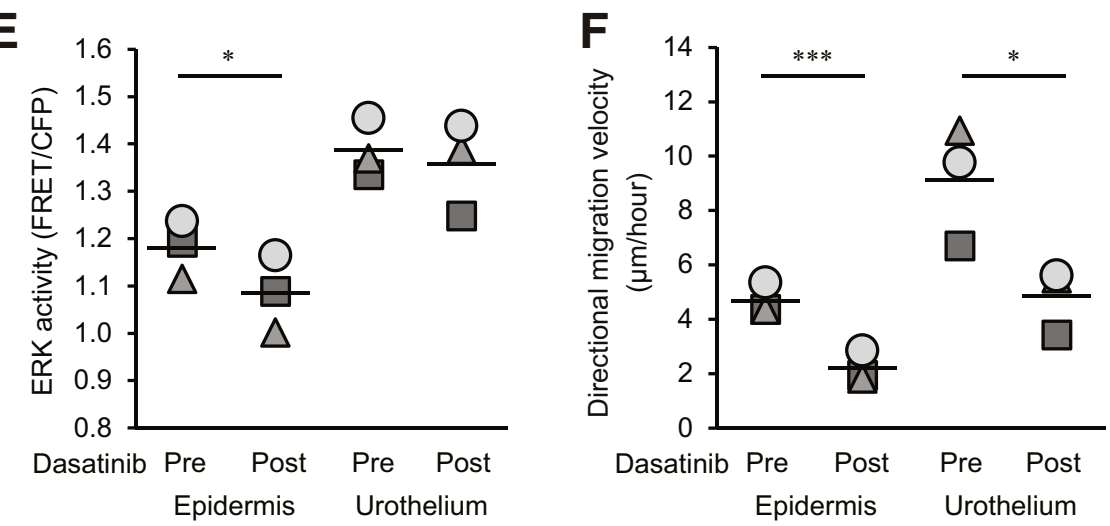$$
\text { bars }=100 \mu \mathrm{m}(\mathrm{A} \text { and } \mathrm{B}) \text {. }
$$

display mode, eight colors from red to blue represent the FRET/CFP ratio, and the 32 grades of intensity represent the signal intensity in each pixel of the CFP image. The warm and cold colors were assigned to high and low FRET levels, respectively. The FRET/CFP ratio of each cell was quantified as follows. For the biosensor located in the nucleus, a region of interest was generated to include each nucleus. For the biosensor located in the cytoplasm, nuclear signals were first subtracted by the H-basin filter of MetaMorph. By using autothreshold, a region of interest was set onto the cytoplasm. Then, the region was expanded three pixels outward. The average fluorescence intensity of the region of interest was used to calculate the FRET/CFP ratio of each cell.

Cell cycle analysis was performed with Fucci mice, according to the method reported previously. ${ }^{17}$ The Fucci biosensor system consisted of two fluorescence reporters, the mKO2-hCdt1 (30/120) $\mathrm{G}_{1}$ marker and mAG-hGeminin $(1 / 110) \mathrm{S} / \mathrm{G}_{2} \mathrm{M}$ marker, which emanate orange and green colors, respectively. For the identification of the nuclei of $\mathrm{S} / \mathrm{G}_{2} / \mathrm{M}$ cells, images of mKO2-hCdt1 (30/120) were subtracted from images of mAG-hGeminin (1/110). The resulting images were processed with the segmentation function of the multidimensional motion analysis module of MetaMorph. The parameters used for the segmentation were as follows: segmentation method, adaptive threshold; $\mathrm{XY}$ diameter, 4 to 20; local intensity above background, 100 . The nuclei of $G_{0} / G_{1}$ cells were identified in a similar manner.

To track cell migration, the FRET images were analyzed by using the Fiji TrackMate plugin. ${ }^{29,30}$ The tracking data were further processed by the Chemotaxis \& Migration Tool version 1.01 (ibidi $\mathrm{GmbH}$, Martinsried, Germany). 


\section{Statistical Analysis}

All statistical analyses were performed using Prism5 software version 6 (GraphPad Software, La Jolla, CA). A paired $t$-test was used to evaluate statistically significant differences. $P<0.05$ was considered statistically significant.

\section{Results}

Time-Lapse Imaging of the Urothelium, the Epidermis, and the Underlying Lamina Propria

Both skin and bladder are covered by stratified epithelium. The remarkable difference between these two epithelia in the mobility over the underlying lamina propria is first shown. Two transgenic mouse strains expressing a nuclear FRET biosensor for ERK, EKAREV-nuclear localization signal, or a cytoplasmic FRET biosensor for ERK, EKAREV-nuclear export signal, were used. ${ }^{25,26}$ Both the epidermis and the urothelium are supported by dense collagen fibers in the lamina propria (Figure 1, A-D). A peculiar anatomic feature of the bladder is the presence of suburothelial capillary plexus and interstitial cells beneath the urothelium (Figure 1, B and D). During the 2-hour observation of mice expressing EKAREV-nuclear localization signal, the nuclei of epidermal basal cells did not move significantly (Figure 1, E and F, and Supplemental Video S1). In stark contrast, the nuclei of urothelial cells were frequently moving (Figure 1, E and $\mathrm{F}$, and Supplemental Video S1). Consequently, the displacement during the 1-hour imaging was larger in the urothelium than the epidermis (Figure 1G). Notably, the urothelial cell sheet occasionally glided over the suburothelial capillary plexus (Figure $1 \mathrm{H}$ and Supplemental Video S2). These observations may suggest that the adhesion to the underlying lamina propria appears markedly weaker in the urothelium than the epidermis. A significant difference was not observed in the mobility between the basal layer cells and the umbrella cells.

\section{Difference in the Mode of Collective Cell Migration} during Wound Healing between the Epidermis and the Urothelium

The seemingly loose adhesion of the urothelium to the underlying lamina propria prompted us to examine the mode of collective cell migration. The epidermis and the urothelium were examined during wound healing by TPEM. In the skin, a microscopic injury of 150 - to $300-\mu$ m diameter was generated with a fine needle, followed by time-lapse imaging (Figure 2A and Supplemental Video S3). Cells were tracked by the TrackMate add-in program in Fiji to calculate their mean velocity and distance from the wound center (Figure 2, B and C). Epidermal cells of two to three rows from the wound edge rapidly migrated toward the wound center, whereas cells behind the front rows migrated rather
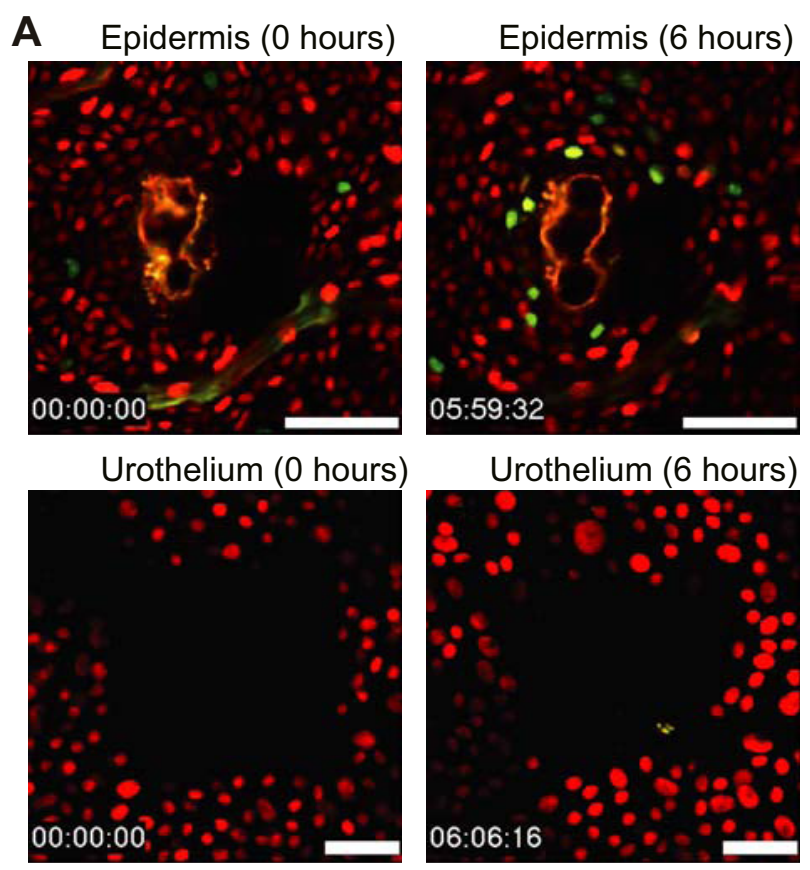

mAG-hGeminin (S/G2/M); mKO2-hCdt1 (G1)

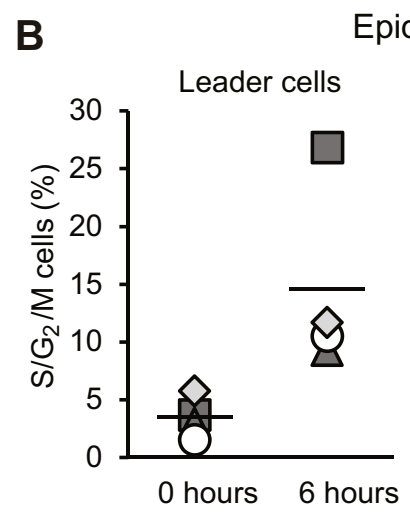

Epidermis

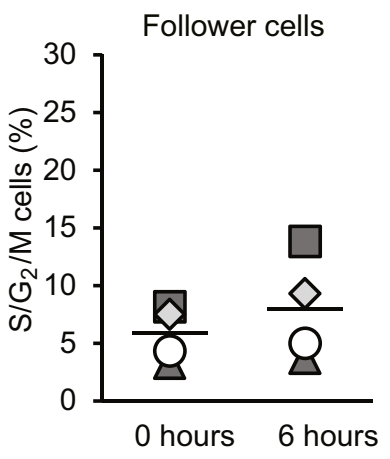

Figure 4 Cell cycle progression in the epidermis but not the urothelium after injury. A: An epithelial wound or a urothelial wound was generated in each transgenic mouse expressing Fucci, a genetically encoded sensor for the cell cycle. The mice were observed by two-photon excitation microscopy for 12 hours. The Fucci biosensor system consisted of an mAG-hGeminin (green) $\mathrm{S} / \mathrm{G}_{2} / \mathrm{M}$ marker and an mK02-hCdt1 (red) $\mathrm{G}_{1}$ marker. B: The percentages of $S / G_{2} / M$ cells in the leader cells, within $20 \mu \mathrm{m}$ of the wound edge, and the other follower cells were scored and plotted. The number of $S / G_{2} / M$ cells in the urothelium was negligible during the observation. $n=4$ (B). Scale bar $=50 \mu \mathrm{m}(\mathbf{A})$.

slowly. This observation agrees with the previously reported results of an in vitro wound healing assay with MDCK cells. ${ }^{31}$ FRET/CFP ratio videos were generated to analyze the dynamics of ERK activity. As reported earlier, ${ }^{17,18}$ ERK activation waves were propagated from the wound edge (Supplemental Video S3).

Similar experiments were set up for the urothelium, although the imaging period of the urothelium could not be as long as that of the ear skin because of the invasiveness of the imaging procedure. Because a mechanical wound could 
Epidermis
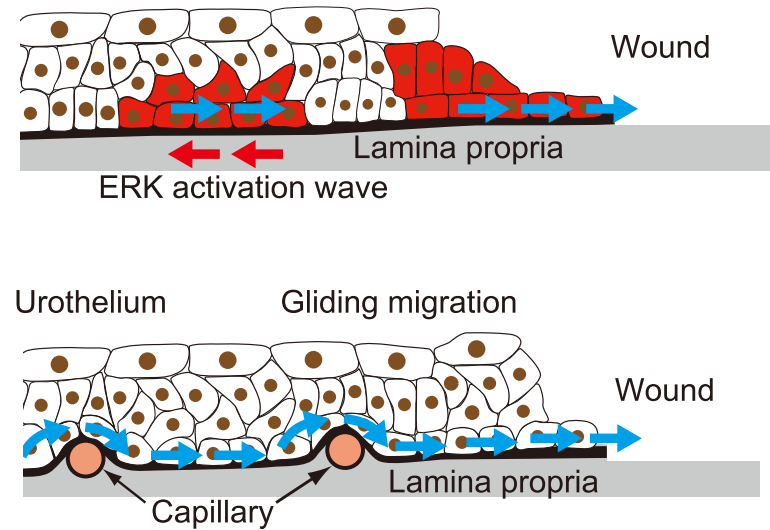

Figure 5 Schematic view of the crawling migration of the epidermis and the gliding migration of the urothelium during the wound healing. In the epidermis, the extracellular signal-regulated kinase (ERK) activation wave is accompanied by a decrease in cell density, which drives the cell sheet to crawl over the underlying lamina propria. In contrast, the urothelium glides over the lamina propria without the waves of ERK activation and cell density change.

not be generated with a fine needle through the urethral catheter, intensive laser radiation was applied to thermally ablate the urothelium. A square $100 \mu \mathrm{m}$ on each side was set on the urothelium and scanned repeatedly under the microscope with $80 \%$ to $100 \%$ laser power until the fluorescence disappeared completely. In this condition, tissue damage of the lamina propria was not detected (Supplemental Figure S1). The wound healing process was initiated soon after the laser ablation (Figure 2, D-F, and Supplemental Video S4). In contrast to the epidermal cells, all urothelial cells within the imaged area glided at similar speeds to fill the defect, indicating that the mode of wound healing is significantly different between the epidermis and the urothelium. In addition, an ERK activation wave was not generated or propagated from the wound edge, suggesting that the biochemical mechanism underlying the cell migration may also be different between the epidermis and the urothelium. The experiments were repeated three times to confirm our observations (Figure 2F). Although the velocity of collective migration changed slightly in each experiment, the mode of collective migration did not change.

\section{Requirement of ERK for the Collective Cell Migration of} the Epidermis but Not the Urothelium

To examine the role of ERK activation in collective cell migration, the MAPK/ERK kinase inhibitor PD0325901 was intravenously administered during time-lapse imaging. By the immunoblotting of the tissue samples, it was confirmed that ERK phosphorylation is markedly suppressed under this condition. ${ }^{25}$ ERK activity and the migration velocity of cells within $20 \mu \mathrm{m}$ of the wound edge were quantitated before and after the inhibitor administration (Figure 3, A-D). In the wounded epidermis, both the basal activity in each cell and the propagation of activation waves of ERK were suppressed by the MAPK/ ERK kinase inhibitor (Figure 3, A and C). Epidermal cell migration was also significantly inhibited, albeit not completely, suggesting the requirement of ERK activity for migration (Figure 3D). In the urothelium as well, PD0325901 inhibited ERK activity (Figure 3, B and C). However, the migration velocity of urothelial cells was not decreased to a statistically significant level, indicating that the ERK activity was dispensable for the gliding migration of the urothelium (Figure 3D).

The Fak-Src-ERK signaling pathway is known to play a pivotal role in the activation and inactivation of integrins at focal contact. ${ }^{32}$ The modest effect of ERK inhibition on the gliding migration of urothelium might suggest that the urothelial cells migrate without disanchoring of the integrin from the underlying matrix. In other words, the urothelium may fill the gap by passive gliding over the lamina propria. To test this hypothesis, the effect of the Src inhibitor dasatinib was examined. The effect of dasatinib on ERK was modest in the epidermis and not significant in the urothelium (Figure 3E). Nevertheless, dasatinib decreased the velocity of not only the epidermis but also the urothelium (Figure 3F). Thus, the urothelial migration, as well as the epidermal migration, requires the activation/ inactivation cycle of integrin-mediated binding to the substrate. This observation also suggests that the urothelial gliding during the wound healing is not a passive movement, but an active tyrosine kinase-dependent cell migration.

\section{Induction of Cell Proliferation in the Epidermis but Not the Urothelium}

Finally, it was examined whether cell proliferation may have any roles in the collective migration of the epidermis and urothelium. The cell cycle was visualized by the use of Fucci mice, in which $\mathrm{G}_{1}$ cells could be discriminated from $\mathrm{S} / \mathrm{G}_{2} / \mathrm{M}$ cells. ${ }^{27}$ To use the same method for the wounding, both the epidermis and the urothelium were ablated in this experiment. Although it took a few hours until the epidermal cells started moving after the laser ablation, collective cell migration was clearly observed. Less than $10 \%$ of basal epidermal cells were in the $S / \mathrm{G}_{2} / \mathrm{M}$ phase before wounding (Figure 4). Six hours after laser ablation, the proportion of $S / G_{2} / M$ cells increased significantly in the regions close to the wound edge, but not in the other regions. In the urothelium, $S / G_{2} / M$ cells were rare, which is consistent with the previous report demonstrating a slow turnover rate $\left(>3\right.$ months). ${ }^{33}$ More important, even after laser ablation, no increase in the proportion of $S / G_{2} / M$ cells was observed. These results strongly suggested that cell proliferation in the epithelium close to the wound may contribute to closing the wound in the epidermis, but not the urothelium, during the time scale of our observation. The 
urothelial cells that entered into the $S / \mathrm{G}_{2} / \mathrm{M}$ phase might be scattered widely in the urothelium outside the viewfield.

\section{Discussion}

For a long period, the urothelium was believed to be pseudostratified and structurally different from the epidermis (ie, all urothelial cells were thought to be more or less connected to the basement membrane). ${ }^{34}$ However, it has been demonstrated that umbrella cells at the apical surface do not have connection to the basement membrane, indicating that the urothelium is a true stratified epithelium like the epidermis. ${ }^{35}$ In this study, in vivo time-lapse imaging clearly demonstrated that the urothelium is more mobile than the epidermis and occasionally glides over the underlying lamina propria, highlighting the significant difference in mobility between the epidermis and the urothelium (Figure 1). This high mobility of the urothelium over the underlying lamina propria was more clearly demonstrated in the collective cell migration during the wound healing process (Figure 2). It can reasonably be speculated that such high mobility, or gliding ability, assists in allowing the urothelium to adapt to the changes in the surface area during the micturition cycle.

It should be emphasized that the gliding of the urothelium over the underlying lamina propria could only be discovered by in vivo time-lapse imaging. To the best of our knowledge, previous observations of the urothelium by TPEM used ex vivo samples. ${ }^{36-38}$ Optimal migration of lymphocytes in explanted lymph nodes requires a high concentration of oxygen. ${ }^{39,40}$ Therefore, the lack of blood flow and resulting low tissue oxygen concentration under the previous experimental conditions might have concealed the gliding of the urothelium. The possibility that the experimental conditions, including anesthesia, also affected the mobility of the urothelium cannot be excluded. At least, the blood flow remained normal under our experimental conditions, ${ }^{25}$ suggesting that our model seems to replicate the physiological conditions in terms of oxygen concentration. Another concern about the in vivo imaging of the bladder is the application of hydrostatic pressure. Currently, the bladder could be observed from the serosa as deep as 350 $\mu \mathrm{m}$ by TPEM. For the acquisition of urothelial images, at least $14 \mathrm{~cm}$ hydrostatic pressure must applied to extend the muscle layer. Therefore, the mode of urothelial cell migration may be different in the bladder at lower intravesical pressure. Last, the epidermis and the urothelium were abraded by needle injection and laser ablation, respectively, to observe the collective cell migration (Figure 2). This is because, in preliminary experiments, the epidermal cells migrated only slowly after the laser ablation, which prevented us from quantitative analysis during the 12-hour time-lapse imaging. Therefore, the difference in the method used to abrade the epithelium might have affected the difference in the mode of migration.
Urothelial cells bind to the basement membrane via hemidesmosomes, as do epidermal cells. ${ }^{41-43}$ Hemidesmosomes are composed of several proteins, among which integrin-family proteins play critical roles to anchor the cells to the basement membrane. ${ }^{44}$ In tissue culture cells, focal adhesions provide the loci for integrinmediated cell-to-substrate anchoring and ERK activity is required for the turnover of the focal adhesions. ${ }^{45,46}$ Taking these previous reports into consideration, the dispensability of ERK for the urothelial migration may suggest the high fluidity of the lamina propria. In other words, the urothelium may be connected only loosely to the underlying lamina propria. However, against this hypothesis, dasatinib, the Src inhibitor, inhibited the urothelial migration (Figure 3F), indicating that the migration of the urothelium is an active process that requires the activation of tyrosine kinases.

In MDCK cells, the ERK activation wave is propagated from the leader cells to promote collective cell migration. ${ }^{10,31}$ More important, the cell density and the ERK activity are inversely correlated. ${ }^{18}$ Therefore, the MDCK cell sheet crawls over the substrate with the cycle of shrinkage and extension of each cell. We speculate that the epidermal cells migrate in a manner similar to MDCK cells (Figure 5). In contrast, the urothelium appears to glide over the lamina propria more smoothly without significant changes in size (Figure 5). This observation may explain the dispensability of ERK activity for the collective cell migration of the urothelium. Inhibitors for the Ras-ERK MAPK pathway are now clinically approved for some cancers, such as melanoma. ${ }^{47}$ Inhibitors against MAPK/ ERK kinase and BRAF are known to perturb the wound healing of the epidermis, ${ }^{48}$ but little is known about the wound healing of the urothelium. Our observations imply that the inhibitors for the Ras-ERK MAPK pathway are less toxic to the urothelium than the epidermis because of the difference in the mode of migration.

In conclusion, in vivo time-lapse imaging of the wound healing process highlighted a marked difference in the mode of the collective cell migration between the epidermis and the urothelium. In vivo TPEM was effective for observing the dynamic nature of cell movement and molecular activities, and its use in these contexts will shed new light on the experimental pathology of wounds.

\section{Acknowledgments}

We thank the members of the Matsuda Laboratory for helpful input, Kyoko Hirano, Kanako Takakura, Nobuyo Sakikawa, and Akiko Kawagishi for technical assistance, and the Medical Research Support Center of Kyoto University for in vivo imaging.

T.S., T.K., O.O., and M.M. conceived the experiments; T.S. performed the experiments; T.S. and M.M. analyzed the data; T.S., T.K., and M.M. wrote the manuscript. 


\section{Supplemental Data}

Supplemental material for this article can be found at https://doi.org/10.1016/j.ajpath.2018.07.010.

\section{References}

1. Rorth P: Collective cell migration. Annu Rev Cell Dev Biol 2009, 25: 407-429

2. Weijer CJ: Collective cell migration in development. J Cell Sci 2009, 122:3215-3223

3. Haeger A, Wolf K, Zegers MM, Friedl P: Collective cell migration: guidance principles and hierarchies. Trends Cell Biol 2015, 25:556-566

4. Martin P: Wound healing: aiming for perfect skin regeneration. Science 1997, 276:75-81

5. Sonnemann KJ, Bement WM: Wound repair: toward understanding and integration of single-cell and multicellular wound responses. Annu Rev Cell Dev Biol 2011, 27:237-263

6. Shaw TJ, Martin P: Wound repair: a showcase for cell plasticity and migration. Curr Opin Cell Biol 2016, 42:29-37

7. Ladoux B, Mege RM, Trepat X: Front-rear polarization by mechanical cues: from single cells to tissues. Trends Cell Biol 2016, 26:420-433

8. Cordeiro JV, Jacinto A: The role of transcription-independent damage signals in the initiation of epithelial wound healing. Nat Rev Mol Cell Biol 2013, 14:249-262

9. Fenteany G, Janmey PA, Stossel TP: Signaling pathways and cell mechanics involved in wound closure by epithelial cell sheets. Curr Biol 2000, 10:831-838

10. Matsubayashi Y, Ebisuya M, Honjoh S, Nishida E: ERK activation propagates in epithelial cell sheets and regulates their migration during wound healing. Curr Biol 2004, 14:731-735

11. Nikolic DL, Boettiger AN, Bar-Sagi D, Carbeck JD, Shvartsman SY: Role of boundary conditions in an experimental model of epithelial wound healing. Am J Physiol Cell Physiol 2006, 291:C68-C75

12. Aoki K, Kamioka Y, Matsuda M: Fluorescence resonance energy transfer imaging of cell signaling from in vitro to in vivo: basis of biosensor construction, live imaging, and image processing. Dev Growth Differ 2013, 55:515-522

13. Oldach L, Zhang J: Genetically encoded fluorescent biosensors for live-cell visualization of protein phosphorylation. Chem Biol 2014, 21 : 186-197

14. Enterina JR, Wu L, Campbell RE: Emerging fluorescent protein technologies. Curr Opin Chem Biol 2015, 27:10-17

15. Kurokawa K, Matsuda M: Localized RhoA activation as a requirement for the induction of membrane ruffling. Mol Biol Cell 2005, 16: 4294-4303

16. Reffay M, Parrini MC, Cochet-Escartin O, Ladoux B, Buguin A, Coscoy S, Amblard F, Camonis J, Silberzan P: Interplay of RhoA and mechanical forces in collective cell migration driven by leader cells. Nat Cell Biol 2014, 16:217-223

17. Hiratsuka T, Fujita Y, Naoki H, Aoki K, Kamioka Y, Matsuda M: Intercellular propagation of extracellular signal-regulated kinase activation revealed by in vivo imaging of mouse skin. Elife 2015, 4: $\mathrm{e} 05178$

18. Aoki K, Kondo Y, Naoki H, Hiratsuka T, Itoh RE, Matsuda M: Propagating wave of ERK activation orients collective cell migration. Dev Cell 2017, 43:305-317

19. Gurtner GC, Werner S, Barrandon Y, Longaker MT: Wound repair and regeneration. Nature (London) 2008, 453:314-321

20. Dalal E, Medalia O, Harari O, Aronson M: Moderate stress protects female mice against bacterial infection of the bladder by eliciting uroepithelial shedding. Infect Immun 1994, 62:5505-5510

21. Veranic P, Jezernik K: The response of junctional complexes to induced desquamation in mouse bladder urothelium. Biol Cell 2000, 92:105-113
22. Apodaca G, Kiss S, Ruiz W, Meyers S, Zeidel M, Birder L: Disruption of bladder epithelium barrier function after spinal cord injury. Am J Physiol Renal Physiol 2003, 284:F966-F976

23. Andreoni CR, Lin HK, Olweny E, Landman J, Lee D, Bostwick D, Clayman RV: Comprehensive evaluation of ureteral healing after electrosurgical endopyelotomy in a porcine model: original report and review of the literature. J Urol 2004, 171:859-869

24. Kreft ME, Sterle M, Veranic P, Jezernik K: Urothelial injuries and the early wound healing response: tight junctions and urothelial cytodifferentiation. Histochem Cell Biol 2005, 123:529-539

25. Sano T, Kobayashi T, Negoro H, Sengiku A, Hiratsuka T, Kamioka Y, Liou LS, Ogawa O, Matsuda M: Intravital imaging of mouse urothelium reveals activation of extracellular signal-regulated kinase by stretch-induced intravesical release of ATP. Physiol Rep 2016, 4: e13033

26. Kamioka Y, Sumiyama K, Mizuno R, Sakai Y, Hirata E, Kiyokawa E, Matsuda M: Live imaging of protein kinase activities in transgenic mice expressing FRET biosensors. Cell Struct Funct 2012, 37:65-73

27. Sakaue-Sawano A, Kurokawa H, Morimura T, Hanyu A, Hama H, Osawa H, Kashiwagi S, Fukami K, Miyata T, Miyoshi H, Imamura T, Ogawa M, Masai H, Miyawaki A: Visualizing spatiotemporal dynamics of multicellular cell-cycle progression. Cell 2008, 132: 487-498

28. Mizuno R, Kamioka Y, Kabashima K, Imajo M, Sumiyama K, Nakasho E, Ito T, Hamazaki Y, Okuchi Y, Sakai Y, Kiyokawa E, Matsuda M: In vivo imaging reveals PKA regulation of ERK activity during neutrophil recruitment to inflamed intestines. J Exp Med 2014, 211:1123-1136

29. Jaqaman K, Loerke D, Mettlen M, Kuwata H, Grinstein S, Schmid SL, Danuser G: Robust single-particle tracking in live-cell time-lapse sequences. Nat Methods 2008, 5:695-702

30. Applegate KT, Besson S, Matov A, Bagonis MH, Jaqaman K, Danuser G: plusTipTracker: quantitative image analysis software for the measurement of microtubule dynamics. J Struct Biol 2011, 176: $168-184$

31. Farooqui R, Fenteany G: Multiple rows of cells behind an epithelial wound edge extend cryptic lamellipodia to collectively drive cell-sheet movement. J Cell Sci 2005, 118:51-63

32. Kleinschmidt EG, Schlaepfer DD: Focal adhesion kinase signaling in unexpected places. Curr Opin Cell Biol 2017, 45:24-30

33. Jost SP: Cell cycle of normal bladder urothelium in developing and adult mice. Virchows Arch B Cell Pathol Incl Mol Pathol 1989, 57: $27-36$

34. Tanaka K: [Polarized light analysis of transitional epithelium in man] German. Arch Histol Jpn 1962, 22:229-236

35. Jost SP, Gosling JA, Dixon JS: The morphology of normal human bladder urothelium. J Anat 1989, 167:103-115

36. Zhuo SM, Chen JX, Luo T, Jiang XS, Xie SS: Multiphoton microscopy of unstained bladder mucosa based on two-photon excited autofluorescence and second harmonic generation. Laser Phys Lett 2009, 6:80-83

37. Xu J, Kang D, Xu M, Zhuo S, Zhu X, Chen J: Multiphoton microscopic imaging of esophagus during the early phase of tumor progression. Scanning 2013, 35:387-391

38. Schueth A, van Zandvoort MA, Buurman WA, van Koeveringe GA: Murine bladder imaging by 2-photon microscopy: an experimental study of morphology. J Urol 2014, 192:973-980

39. Miller MJ, Wei SH, Parker I, Cahalan MD: Two-photon imaging of lymphocyte motility and antigen response in intact lymph node. Science 2002, 296:1869

40. Huang JH, Cárdenas-Navia LI, Caldwell CC, Plumb TJ, Radu CG, Rocha PN, Wilder T, Bromberg JS, Cronstein BN, Sitkovsky M, Dewhirst MW, Dustin ML: Requirements for T lymphocyte migration in explanted lymph nodes. J Immunol 2007, 178:7747

41. Alroy J, Gould VE: Epithelial-stromal interface in normal and neoplastic human bladder epithelium. Ultrastruct Pathol 1980, 1: 201-210 
42. Woldemeskel M, Drommer W, Wendt M: Histology and ultrastructure of the urothelium lining the ureter and the renal pelvis in sows. Anat Histol Embryol 1998, 27:51-55

43. Owaribe K, Kartenbeck J, Stumpp S, Magin TM, Krieg T, Diaz LA, Franke WW: The hemidesmosomal plaque, I: characterization of a major constituent protein as a differentiation marker for certain forms of epithelia. Differentiation 1990, 45:207-220

44. Parsons JT, Horwitz AR, Schwartz MA: Cell adhesion: integrating cytoskeletal dynamics and cellular tension. Nat Rev Mol Cell Biol 2010, 11:633-643

45. Xie H, Pallero MA, Gupta K, Chang P, Ware MF, Witke W, Kwiatkowski DJ, Lauffenburger DA, Murphy-Ullrich JE, Wells A: EGF receptor regulation of cell motility: EGF induces disassembly of focal adhesions independently of the motility-associated $\mathrm{PLC} \gamma$ signaling pathway. J Cell Sci 1998, 111:615-624

46. Fincham VJ, James M, Frame MC, Winder SJ: Active ERK/MAP kinase is targeted to newly forming cell-matrix adhesions by integrin engagement and v-Src. EMBO J 2000, 19:2911-2923

47. Simanshu DK, Nissley DV, McCormick F: RAS proteins and their regulators in human disease. Cell 2017, 170:17-33

48. Escuin-Ordinas H, Li S, Xie MW, Sun L, Hugo W, Huang RR, Jiao J, de-Faria FM, Realegeno S, Krystofinski P, Azhdam A, Komenan SMD, Atefi M, Comin-Anduix B, Pellegrini M, Cochran AJ, Modlin RL, Herschman HR, Lo RS, McBride WH, Segura T, Ribas A: Cutaneous wound healing through paradoxical MAPK activation by BRAF inhibitors. Nat Commun 2016, 7:12348 